696 Hans FrtTing: Beobachtungen über die Induktion der Dorsiventralität.

Es sieht auch so aus, als ob zwischen der Entwicklung der Spaltöffnungen und des Mesophylls (einschließlich des Sklerenchyms) eine enge Korrelation bestünde, deren Ursachen sich noch gar nicht durchschauen lassen. Erst die fernere Zukunft dürfte hier vielleicht zu einer gewissen Einsicht verhelfen.

Die bisherigen Versuche, die eingehender ja nur bei Thujopsis dolabrata durchgeführt worden sind, bedürfen nach manchen Richtungen noch weiterer Ergänzungen. Selbstverständlich eignen sich däür nux solche Arten, bei denen durch die einseitige Beleuchtung ähnlich ausgesprochen dorsiventraler Bau der Zweige hervorgerufen wird wie bei dieser Art. Dies aber ist der Fall unter den von mir anatomisch näher untersuchten Cupressaceen bei Libocedrus macrolepis, Thuja occidentalis, Th. gigantea, Th. Standishii sowie bei Chamaecyparis pisifera. Wichtig wäre es dazu vor allem, wenn sich weitere Arten finden ließen, deren Zweigstecklinge sich zu bewurzeln vermögen, oder deren Keimlinge für solche Versuche sich eignen. Während Stecklinge sich trotz manchen Bemühungen bei Libocedrus macrolepis (und $L$. decurrens) nicht bewurzelten, berechtigen die anderen genannten Arten auch nach gärtnerischen Erfahrungen in dieser Hinsicht immerhin zu einigen Hoffnungen.

\title{
Literatur.
}

Beissner, L.: Handbuch der Koniferenbenennung. Erfurt 1887. - Handbuch der Nadelholzkunde. Berlin 1891. - Eichler, A. W.: In Engler u. Prante, Natürliche Pflanzenfamilien, 1.Aufl., Bd.2. Leipzig 1889. - Fitriva, H.: Über die Induktion der Dorsiventralitât in den blattähnlichen Zweigsystemen der Cupressaceen. Jb. Bot. 90, 417 (1942). - FraNK, A. B.: Über den Einfluß des Lichtes auf den bilateralen Bau der symmetrischen $Z$ weige der Thuja occidentalis. Jb. Bot. 9, 147 (1873/74). - ImamuRa, SH.: Über die aitiogene Dorsiventralität der Assimilationsorgane bei höheren Pflanzen. Bot. Mag. 51, 490 (1937). - KLEMM, P.: Ü̉ber den Bau der beblätterten Zweige der Cupressineen. Jb. Bot. 17, 499 (1886). - KUGLER, H.: Über invers-dorsiventrale Blätter. Planta (Berl.) 5, 89 (1928). - Pick, H.: Über den Einfluß des Lichtes auf die Gestalt und Orientierung der Zellen des Assimilationsgewebes. Bot. Zbl. 11, 400 (1882). - Pilger, R.: In Engler u. Prajte, Natürliche Pflanzenfamilien, 2. Aufl., Bd. 13. Leipzig 1926. - Trour, W.: Vergleichende Morphologie der höheren Pflanzen, Bd. 1/2. Berlin 1939.

Prof. Dr. Hans Fitting, Bonn, Am Berghang 6.

\section{Berichtigung zu Band 36, Heft $\mathbf{5} / 6$.}

Beitrag TRoLL ,Über Leitbündelisolierung in achsenartigen Organen“. Auf S. 408 sind in Abb. 6 die Teilfiguren III und IV verwechselt worden; III (also eigentlich IV) steht außerdem auf dem Kopf. Das gleiche gilt für $A b b .5 I I$ auf S. 407. 